\title{
3 Research Square

\section{Gaps in patient-physician communication at the time of malignant bowel obstruction from recurrent gynecologic cancer: a qualitative study.}

Claire Hoppenot ( $\sim$ claire.hoppenot@gmail.com )

Baylor College of Medicine https://orcid.org/0000-0003-0941-6689

\section{Fay J Hlubocky}

University of Chicago Hospital: The University of Chicago Medicine

Julie Chor

University of Chicago Hospital: The University of Chicago Medicine

\section{S. Diane Yamada}

University of Chicago Hospital: The University of Chicago Medicine

\section{Nita Lee}

University of Chicago Hospital: The University of Chicago Medicine

\section{Research Article}

Keywords: Malignant bowel obstruction, patient-physician communication, palliative care, qualitative research, gynecologic cancer

Posted Date: March 29th, 2021

DOI: https://doi.org/10.21203/rs.3.rs-298194/v1

License: (c) (i) This work is licensed under a Creative Commons Attribution 4.0 International License. Read Full License

Version of Record: A version of this preprint was published at Supportive Care in Cancer on July 21st, 2021. See the published version at https://doi.org/10.1007/s00520-021-06441-0. 


\section{Abstract}

Purpose We sought to investigate the patient and physician approaches to malignant bowel obstruction (MBO) due to recurrent gynecologic cancer by 1) comparing patient and physician expectations and priorities during a new MBO diagnosis, and 2) highlighting factors that facilitate patient-doctor communication.

Methods Patients were interviewed about their experience during an admission for $\mathrm{MBO}$, and physicians were interviewed about their general approach towards MBO. Interviews were analyzed for themes using QDAMiner qualitative analysis software. The analysis utilized the framework analysis and used both predetermined themes and those that emerged from the data.

Results We interviewed 14 patients admitted with MBO from recurrent gynecologic cancer and 15 gynecologic oncologists. We found differences between patients and physicians regarding plans for next chemotherapy treatments, foremost priorities, communication styles, and end-of-life discussions. Both patients and physicians felt that the patient-physician communication was improved in situations of trust, understanding patient preferences, corroboration of information, and increased time spent with patients during and before the MBO.

Conclusion Gaps in patient-physician communication could be targeted to improve the patient experience and physician counseling during a difficulty diagnosis by focusing on education, symptoms, home support, nutrition, and end-of-life care.

\section{Introduction}

A malignant bowel obstruction (MBO) is a turning point in the care of women with a gynecologic cancer. From the patient perspective, MBO is associated with many distressing symptoms including nausea, vomiting, pain, and inability to eat that are difficult to control for many women who may have been previously only mildly symptomatic during initial treatment for recurrent disease. Physicians reevaluate whether to stop previously established treatment plans, including chemotherapy, until MBO resolution or indefinitely.

Literature on $\mathrm{MBO}$ prognosis and interventions shows a median survival after $\mathrm{MBO}$ ranging between one and six months [1] [2] [3] [4] [5]. However, qualitative studies have shown that it is not always clear to ovarian cancer patients that a MBO is a turning point of disease trajectory and prognosis [6]. There is a gap between the medical data available and the patient understanding of MBO. Studies in settings other than MBO have shown that although oncologists report discussing prognosis or palliative intent of interventions such as chemotherapy, at least one third of patients were unable to reflect that understanding [7]. Communication strategies have focused on how to bridge such gaps. For example, qualitative studies have shown that patients prefer serious news disclosures to occur in quiet places, oneon-one, with a physician who can give a comprehensive evaluation of the diagnosis, prognosis, and 
treatment options [7]. In transitions from curative to palliative care, patients have highlighted the importance of clarity of information, grieving loss, and sustaining hope [7] [8].

In this qualitative study, our objective was to explore physician and patient approaches to communication and treatment management for the diagnosis of MBO. We interviewed patients during the MBO admission at two academic-affiliated hospitals and gynecologic oncologists who frequently cared for women with MBO at these and other hospitals. Our goals were to 1) compare patient and physician expectations and priorities during a new MBO diagnosis, and 2) highlight factors noted by patients and physicians to facilitate patient-doctor communication.

\section{Methods}

Patient interviews were conducted at two institutions (the University of Chicago and Northshore University Health System). The patient interviews were granted IRB approval and cancer center approval at both institutions at which the interviews were conducted, and the physician interviews were granted an IRB exemption through the University of Chicago. The patient census was followed and those admitted for a MBO between $5 / 2016$ and 10/2018 were flagged by the primary researcher $(\mathrm{CH})$. The primary researcher was a research fellow. During her clinical years, she was involved in the care of the last three patients interviewed, and worked with seven of the interviewed gynecologic oncologists.

Inclusion criteria included malignant bowel obstruction based on clinical findings and/or imaging, known recurrent or progressive gynecologic with previous treatment, age 18-89, English-speaking, and able and willing to participate in an interview. Patients considering hospice after discharge from the hospital were included. The charts were reviewed and after discussion with the primary team to ensure the patient was a suitable candidate, she was approached by the researcher regarding participation in the study to help describe her experience with MBO. If the patient agreed, consent was signed and the semi-structured interview was performed in person and recorded by a single interviewer $(\mathrm{CH})$. The timing of all interviews was one to two days prior to anticipated discharge, after most of the decision-making and counseling had occurred. If patients were readmitted during the study period, they could be re-interviewed regarding their post-discharge experience. All interviews were recorded and transcribed, with all patient identifiers and treating institutions removed prior to analysis.

For physician interviews, a list of local gynecologic oncologists was collected from a department contact list. A standardized email was sent to 27 gynecologic oncologists and a positive response was considered consent to participate. Semi-structured interviews were then scheduled and conducted via phone or in person by a single interviewer $(\mathrm{CH})$. After obtaining oral consent, interviews were digitally recorded and subsequently transcribed, either by the interviewer or professionally. After transcription, all identifiers were removed from transcripts.

The interview guides were created after a literature review and follow-up questions were revised iteratively in response to the focus of previous interviews (Suppl 1). The patient interview guide was designed to assess patient symptoms and needs during admission for MBO, decisions made during the admission as 
well as since diagnosis, communication with her doctor, and plans after discharge. The physician guide was designed to explore the physicians' approach to care of patients admitted with MBO from recurrent or progressive gynecologic cancer. Questions addressed standard management practices, patient concerns, comfort with end of life discussions, and personal toll of managing MBO. The backbone of the interview guides did not change over the course of the interviews but follow up questions and clarifications were adjusted based on each individual interview to allow interviewees to highlight areas that they prioritized.

\section{Data analysis:}

Interviews were recorded and transcribed. QDAMiner qualitative analysis software (Provalis Research, Montreal, Canada) was used to organize, store and compare notes. The interviews were then systematically analyzed for themes using the framework analysis, which uses a constant comparative technique across interviews to refine themes as new data is analyzed throughout the process [9] [10] [11]. Framework analysis uses both deductive codes and themes, predetermined by prior research, and open coding, which is developed and refined from the data itself, and these themes are then organized and connections are created based on the data abstracted from interviews to develop an overarching theory [12] [9] [10] [11].

Predetermined objectives included (1) a comparison of the priorities of physicians and patients regarding symptom control, home support, and end of life concerns, (2) description of aspects of patient-doctor communication during the time of MBO that functioned well, and (3) eliciting communication strategies from physicians. The first 3-4 interviews were coded immediately to help inform subsequent interviews on areas requiring clarification, and analysis continued throughout the interviews to establish when theme saturation was reached. Data dictionaries were developed for the patient and physician interviews and iteratively revised by 2 co-investigators ( $\mathrm{CH}$ and $\mathrm{NL}$ for physician interviews, $\mathrm{CH}$ and $\mathrm{FH}$ for patient interviews). Codes were reviewed for concordance and differences in coding were resolved by mutual agreement. While there was overlap in the data dictionaries, creating different dictionaries for patients and physicians provided more flexibility to highlight unique aspects of each interviewed group.

The total number of interviews required was determined by theme saturation. Once interviews stopped providing new themes or adding to the theories developed, no further interviews were conducted.

Thematic saturation was reached after 11-13 interviews for both patients and physicians, and 2-3 further interviews were conducted for each group to ensure that no further themes emerged.

\section{Results}

Twenty patients were approached for interviews, and 14 agreed to proceed (Table 1a). The most common reason for refusal was not feeling well enough for the interview. Five of the six patients who declined to be interviewed identified as Black. More than half of patients who agreed to participate identified as white $(53 \%)$ and most had ovarian/peritoneal cancer. Age ranged from 36 to 81 years of age. Eleven patients were married. Thirteen participants were interviewed at the time of a first admission for MBO (PT 2 had a 
remote history of an MBO). PT 5 was readmitted and had surgery days after her first discharge; information from a second interview was combined with the first interview. Interview length ranged from 20 minutes to 90 minutes.

For the physician interviews, 27 physicians were contacted via a standardized email. Absence of response was followed up with an attempt at contact by phone. Six were never reachable, one responded and declined participation, five were willing but unable to schedule the interview, and 15 gynecologic oncologists were interviewed (Table 1b). All 15 gynecologic oncologists provided both surgery and chemotherapy for their patients.

\section{Discrepancies in approaches and priorities}

Several differences emerged in the patient experience and physician approaches to MBO (see Table 2 for select quotations). These can be categorized as differences in patient goals, expectation for end-of-life conversations, ability to communicate information, communication style, and decision-making.

In discussing their goals, patients repeatedly mentioned a next, future line of chemotherapy. Physicians, however, emphasized the fact that an MBO was an end-of-life diagnosis. Additionally, when physicians were asked what they believed were patients' primary concerns at the time of MBO, almost all reported nutrition as foremost. Participating patients did mention nutrition but tended to emphasize the benefits of being at home.

Patients had a wide range of responses to questions regarding end-of-life goals. In particular, young patients with small children (PT 6, PT 9) had the shortest interviews and focused on next treatments and survival. They were not ready to discuss end-of-life planning. On the other hand, some patients who were diagnosed 5-10 years before their interview (such as PT 2, PT 10 and PT 14) seemed to accept the diagnosis and had specific goals of care. Almost all participating physicians described the importance of end-of-life and goals-of-care conversations with patients admitted for MBO.

Participants also brought up several differences in communication. The amount of information was a frequent theme; many patients voiced frustration with the paucity of information they received, while physicians struggled with how to prognosticate and provide counseling in the setting of so much data and uncertainty. In terms of style, patients valued positive thinking, while many physicians were worried about keeping expectations realistic.

Lastly, the experience of decision-making was very different for patients and physicians. Patient participants were asked specifically what decisions they had made about their care during their hospitalization; most patients denied having made any. Many commented that they deferred to their physicians' recommendations, and some even reported hoping they would not have to make decisions. Participating physicians wrestled with the pros and cons of medical managements as well as whether to take a patient to surgery. They noted that the amount of uncertainty about risks and benefits of all options made counseling and decision-making difficult at the time of MBO. 
Patients and physicians did agree on many factors that positively influenced patient-doctor communication at the time of MBO diagnosis (see Table 3 for select quotations). First, a patient's trust of her physician was a common factor that allowed patients to understand and accept the diagnosis. Second, both patients and physicians reported that patients "being and feeling heard" improved a physician's ability to counsel the patient. The responsibility was not only on the physicians; some patients blamed themselves for not expressing themselves clearly or loudly enough, and when asked what they recommended to other women in their situation, many patient participants suggested speaking up more for themselves. Third, patients and physicians valued corroboration of information and validation of decision-making. For patients, this took the form of second opinions, personal research, and discussions with family. Physicians consulted other services (most commonly general surgery or palliative care) and stressed the importance of discussing MBO patients with colleagues. Lastly, both patients and physicians noted that time was important; both time for counseling during the acute MBO episode, and the length of time to develop a relationship with the patient prior to their admission for MBO.

Time with the patient did prove to be a double-edged sword for some physicians: although a long-term relationship with the patient could make conversations easier, it came with more personal sadness and distress during goals of care discussion. Some physicians recognized that this closeness might also introduce bias into the decision-making process.

\section{Communication strategies}

The participating physicians noted multiple communication strategies for difficult conversations (Table 4). The strategies depended on physician preferences, but many also reported adapting their approach to individual clinical situations. Physicians overall were divided between offering options and always having a more directed recommendation. When multiple options appeared equivalent, they were more likely to offer options. At the same time, many physicians felt strongly that it was their professional duty as a physician to provide a recommendation. Overall, they did not find that providing statistical data was helpful to assist patient decisions, but some used best- and worse-case scenarios to put potential outcomes in perspective.

\section{Discussion}

An MBO diagnosis is devastating for many patients, and is also challenging personally and professionally for the physicians caring for them. It results in high symptom burden and need for increased supportive care, and forces a reevaluation of the treatment plan, an acknowledgement of an uncertain prognosis, and an exploration of end-of-life preferences. This qualitative study highlighted some differences in the priorities and concerns brought up by patients and gynecologic oncologists regarding MBO. We hope that acknowledging these differences can help physicians lead more patientcentered discussions. 
The widest gap between the patients and physicians approaches to MBO was in expectations for future treatments. A primary priority for many patients was to get back on chemotherapy, and at the time of discharge, almost all interviewed patients reported plans to have more chemotherapy. Yet the broadsweeping approach of the participating physicians was that chemotherapy after MBO was unlikely to be beneficial. This discrepancy may in part be explained by the fact that almost all participating patients were diagnosed with their first MBO episode, and still had good performance status and symptom control. Only one participant was discharged with hospice. In fact, retrospective studies have found that after a first diagnosis of MBO from recurrent ovarian or uterine cancer, almost half of patients had subsequent chemotherapy $[1,13]$. The difference between the reported physician counseling and studies showing chemotherapy use suggests that either physicians act differently than they admit in interviews, or that when physicians reflect about MBO, the most memorable discussions are those with patients transitioning to hospice, not the counseling at the first MBO. It may be helpful for physicians to acknowledge this bias when preparing to counsel a patient with MBO regarding future options and expectations.

Patient participants focused on their desire to be home, in parallel with concerns about being able to manage symptoms at home. Fewer physician interviewees addressed this issue. Programs focusing on outpatient follow-up of patients with MBO have been able to support patients in their desire to be at home, as well as addressing issues of nutrition. Lee et al provided patients with education on MBO symptoms and diagnosis, as well as frequent check-ins after discharge home [14]. A qualitative analysis showed that patients valued these interventions, and their daily concerns and changes in symptoms could be managed outpatient by an expert team, to prioritize their ability to be home [6].

Patients' worry about acquiring information manifested in two ways: concern about physician communication and, more commonly, frustration at diagnostic or prognostic uncertainty of their MBO. Uncertainty is a common concern for cancer patients, and it has previously been associated with lower quality of life throughout the course of cancer treatment [15]. Participating physicians also struggled with managing uncertainty in counseling and discussion, and uncertainty is a known barrier to difficult conversations [16]. Studies suggest providing patient education can counteract some of the negative effects of prognostic uncertainty [17], but participating physicians did not find that providing statistical data was helpful for patients. Determining how to provide patient-centered information about MBO may help bridge this communication gap between patients and physicians.

Some physicians used best case-worst case scenarios in their discussions. Surgeon and ethicist Margaret Schwarze suggests this as a strategy to communicate with patients in the face of uncertainty. She describes using best and worst case scenarios related to a patient's diagnosis or upcoming procedure [18]. Rather than depend on statistics, the scenarios provide anchors for likely and possible outcomes, giving a range and managing expectations about whether returning to a prior baseline is an option. Scenarios allow a description of what is known, and what is uncertain. It allows personalization to a patient's specific case and preferences. In light of the physicians' concerns over use of numerical data, best-worst case scenarios can provide patient education without being overwhelming. 
Physician participants were asked about communication strategies, and none reported using guides or decision aids. Yet a structured communication tool could address many of the struggles they and patients describe, including managing uncertainty and communicating overwhelming quantities of information. In a Cochrane review of 105 studies evaluating patients facing treatment and screening decisions, decision aids helped patients have a more active role in decision-making [19]. They also helped patients feel better informed about their diagnosis and their values. The average consultation length was not increased. Decision aids have been evaluated in the setting of suboptimally debulked ovarian cancer, and were found to successfully provide patient education [20], but no decision aids have been made for $\mathrm{MBO}$ in the literature.

In a review of decision aids based on shared decision-making, Wieringa et a/ define 6 key elements of a decision aid: situation/diagnosis, choice awareness, option clarification, harms and benefits discussion, patient preference deliberation, and making a decision [21]. While a "decision aid" may not be appropriate in a setting such as MBO where many patients did not remember making decisions, a structured communication guide for physicians could help bridge communication gaps highlighted in our interviews (Table 5). We would suggest a guide using elements of Weiringa et a/s decision aids in combination with facilitators and discrepancies elicited during patient and physician interviews. Ideally, it would prompt physicians and patients to better define realistic patient goals. In particular, many of our participants wanted to go home; eliciting home needs and providing follow-up could help empower patients after discharge. Uncontrolled symptoms and a plan for worsening of symptoms should be discussed. It should address the possibility of future treatments, and events that would require stopping chemotherapy.

As was previously published, most participating gynecologic oncologists feel comfortable having these goal-of-care discussions, although they are frequently conducted in conjunction with palliative care [22]. Almost all participating physicians reported planning end-of-life conversations for patients with $\mathrm{MBO}$, but not all patients were ready for that discussion. It is a difficult conversation. Training during and after fellowship can help support physicians through the process. Tips from well validated and accessible communication skills programs such as Vital Talk, for example, can provide strategies to address difficult aspects of the conversation (https://www.vitaltalk.org/clinicians/). Our study highlights the particular difficulty in addressing these concerns for recently-diagnosed, young patients, and early palliative care referrals in particular for these patients may help provide support for physicians and patients in planning for end-of-life care.

Our study is not without limitations. Both physicians and patients were limited to a single urban Midwest geographic area. Additionally, most patients who agreed to the interview were admitted for their first admission for MBO and had good symptom control; they may not be as representative of patients with more advanced or recurrent MBO. Only women with gynecologic cancer were included in this study, and they may not be representative of men and women with other cancer histologies, or at other time courses in their disease. It is unknown whether patients who declined to participate in the interviews would have provided different insight. Physicians were questioned in general terms about patients with MBO, while participating patients were focused on their individual experience, which can limit the comparisons 
between the two interview groups. As a qualitative study, it is designed to bring further questions that can be explored. Other perspectives that would be interesting to investigate include those of bedside nursing, family members, home health, and palliative care/hospice teams.

Our study's strengths include the ability to interview both patients and gynecologic oncologists to address different perspectives of the challenging topic. The interviews were conducted by one interviewer for consistency, and she was not clinically involved in the majority of the care of the patients interviewed. The framework method allowed us to start with themes from the literature while simultaneously developing codes and themes coming from the interviews themselves.

\section{Conclusion}

Interviews with women with MBO and gynecologic oncologists who care for women with MBO revealed discrepancies in approach between the two groups, but also factors that facilitate the patient-physician communication that can be further developed. A patient-centered communication guide for physicians and patients may be helpful to trigger discussions about aspects of care that patients highlighted, such as nutrition and timing to discharge home. It could also leave patients feeling better informed in the face of uncertainty and physicians better supported to provide information, which were themes in both groups of interviewees. Further research to develop a formal communication aid and to tailor it to specific patient and physician populations and hospital resources is needed.

\section{Declarations}

Funding: Partial financial support was received from Bears Care, the charitable arm of the Chicago Bears Football Club (CH, SDY).

Conflicts of Interest/Competing interests: The authors have no conflicts of interest to report.

Availability of data and material: Not applicable

Code availability: Not applicable

Author contributions: $\mathrm{CH}, \mathrm{FH}, \mathrm{JC}, \mathrm{SDY}$ and $\mathrm{NL}$ all contributed to the project development and study design. $\mathrm{CH}$ conducted the interviews and some of the transcription. $\mathrm{CH}, \mathrm{FH}$ and $\mathrm{NL}$ shaped the data dictionary and analyzed the transcripts. $\mathrm{CH}, \mathrm{FH}, \mathrm{JC}, \mathrm{SDY}$ and $\mathrm{NL}$ contributed to writing the manuscript.

Ethics approval: The patient interviews were approved by the IRBs of the University of Chicago and Northshore University Health System. Physician interviews were granted IRB exemption by the University of Chicago.

Consent to participate: Patients signed a standard IRB-approved consent prior to participation, which included agreeing to audio recording. Physician consent was confirmed by a positive response to a standard email, and verbal consent was elicited for audio recordings. 
Consent for publication: Patients consented to publishing of the findings from the study.

\section{References}

[1] Hoppenot C, Peters P, Cowan M, Moore ED, Hurteau J, Lee NK, et al. Malignant bowel obstruction due to uterine or ovarian cancer: Are there differences in outcome? Gynecologic oncology. 2019;154:177-82.

[2] Lilley EJ, Scott JW, Goldberg JE, Cauley CE, Temel JS, Epstein AS, et al. Survival, Healthcare Utilization, and End-of-life Care Among Older Adults With Malignancy-associated Bowel Obstruction: Comparative Study of Surgery, Venting Gastrostomy, or Medical Management. Ann Surg. 2017.

[3] Mooney SJ, Winner M, Hershman DL, Wright JD, Feingold DL, Allendorf JD, et al. Bowel obstruction in elderly ovarian cancer patients: a population-based study. Gynecologic oncology. 2013;129:107-12.

[4] Perri T, Korach J, Ben-Baruch G, Jakobson-Setton A, Ben-David Hogen L, Kalfon S, et al. Bowel obstruction in recurrent gynecologic malignancies: defining who will benefit from surgical intervention. Eur J Surg Oncol. 2014;40:899-904.

[5] Terrah J Paul Olson CP, Karen J Brasel, Margaret L Schwarze. Palliative Surgery for malignant bowel obstruction from carcinomatosis, a systematic review. JAMA. 2014;149:383-92.

[6] Cusimano MC, Sajewycz K, Nelson M, Jivraj N, Lee YC, Bowering V, et al. Supported self-management as a model for end-of-life care in the setting of malignant bowel obstruction: A qualitative study. Gynecologic oncology. 2020.

[7] Back A, Anderson WG, Bunch L, Marr LA, Wallace J, Yang H, et al. Communication about cancer near the end of life. Cancer. 2008;113:1897-910.

[8] Hagerty R, Butow P, Ellis P, Lobb E, Pendlebury S, Leighl N, et al. Communicating with realism and hope: incurable cancer patients' views on the disclosure of prognosis. J Clin Oncol. 2005:1278-88.

[9] Mays N, Pope C. Qualitative research in health care. Assessing quality in qualitative research. BMJ. 2000;320:50-2.

[10] Pope C, Ziebland S, Mays N. Qualitative research in health care. Analysing qualitative data. BMJ. 2000;320:114-6.

[11] Ritchie JSL. Qualitative data analysis for applied policy research. London: Routledge; 1994.

[12] Mays N, Pope C. Qualitative research: Observational methods in health care settings. BMJ. 1995;311:182-4.

[13] Diver E, O'Connor O, Garrett L, Boruta D, Goodman A, Del Carmen M, et al. Modest benefit of total parenteral nutrition and chemotherapy after venting gastrostomy tube placement. Gynecologic oncology. 
[14] Lee YC, Jivraj N, Wang L, Chawla T, Lau J, Croke J, et al. Optimizing the Care of Malignant Bowel Obstruction in Patients With Advanced Gynecologic Cancer. J Oncol Pract. 2019:JOP1800793.

[15] Ghodraty Jabloo V, Alibhai SMH, Fitch M, Tourangeau AE, Ayala AP, Puts MTE. Antecedents and Outcomes of Uncertainty in Older Adults With Cancer: A Scoping Review of the Literature. Oncol Nurs Forum. 2017;44:E152-E67.

[16] Bernacki RE, Block SD, American College of Physicians High Value Care Task F. Communication about serious illness care goals: a review and synthesis of best practices. JAMA Intern Med. 2014;174:1994-2003.

[17] Dean M, Davidson LG. Previvors' Uncertainty Management Strategies for Hereditary Breast and Ovarian Cancer. Health Commun. 2018;33:122-30.

[18] Schwarze ML, Taylor LJ. Managing Uncertainty - Harnessing the Power of Scenario Planning. N Engl J Med. 2017;377:206-8.

[19] Stacey D, Legare F, Lewis K, Barry MJ, Bennett CL, Eden KB, et al. Decision aids for people facing health treatment or screening decisions. Cochrane Database Syst Rev. 2017;4:CD001431.

[20] Elit LM, Levine MN, Gafni A, Whelan TJ, Doig G, Streiner DL, et al. Patients' preferences for therapy in advanced epithelial ovarian cancer: development, testing, and application of a bedside decision instrument. Gynecologic oncology. 1996;62:329-35.

[21] Wieringa TH, Rodriguez-Gutierrez R, Spencer-Bonilla G, de Wit M, Ponce OJ, Sanchez-Herrera MF, et al. Decision aids that facilitate elements of shared decision making in chronic illnesses: a systematic review. Syst Rev. 2019;8:121.

[22] Hoppenot C, Hlubocky FJ, Chor J, Yamada SD, Lee NK. Approach to Palliative Care Consultation for Patients With Malignant Bowel Obstruction in Gynecologic Oncology: A Qualitative Analysis of Physician Perspectives. JCO Oncol Pract. 2020:JOP1900710.

\section{Tables}

Table 1a: Characteristics of participating patients admitted for MBO from recurrent/progressive gynecologic cancer $(\mathrm{n}=14)$ 


\begin{tabular}{|c|c|c|c|c|c|c|}
\hline ID\# & Age $^{1}$ & Race $^{2}$ & Religion $^{2}$ & Cancer site $^{1}$ & Interventions $^{1}$ & - discharge plan ${ }^{1}$ \\
\hline 1 & 69 & White & Catholic & fallopian tube & duodenal stent & chemo \\
\hline 2 & 68 & White & Catholic & endometrium & medical $^{3}$ & chemo \\
\hline 3 & 46 & Hispanic & Catholic & ovary & medical $^{3}$ & chemo \\
\hline 4 & 68 & White & None & ovary & medical $^{3}$ & chemo \\
\hline 5 & 63 & White & Catholic & ovary & surgery (diverting ostomy) & clinical trial \\
\hline 6 & 36 & Black & None & cervix & medical $^{3}$ & chemo \\
\hline 7 & 57 & Black & Baptist & cervix & surgery (diverting ostomy) & chemo \\
\hline 8 & 60 & Hispanic & Christian & ovary & medical $^{3}$ & chemo \\
\hline 9 & 43 & White & None & ovary & medical $^{3}$ & clinical trial \\
\hline 10 & 55 & White & Catholic & ovary & medical $^{3}$ & chemo \\
\hline 11 & 69 & Black & Catholic & cervix & medical $^{3}$ & consider surgery \\
\hline 12 & 81 & Black & Christian & ovary & medical $^{3}$ & chemo \\
\hline 14 & 62 & White & None & ovary & percutaneous gastrostomy tube & hospice \\
\hline 15 & 54 & White & Jewish & peritoneum & percutaneous gastrostomy tube & second opinion \\
\hline
\end{tabular}

1 Collected from the medical record

2 Self-identified

3 Patients treated with medications for symptom control, with or without nasogastric tube placement, without further intervention.

\begin{tabular}{lc}
\hline Table 1b: Characteristics of participating gynecologic oncologists $(\mathbf{n}=\mathbf{1 5})$ \\
\hline Gender & $8(53 \%)$ \\
$\quad$ Female & $7(47 \%)$ \\
\hline Male & $10(67 \%)$ \\
\hline Practice type & $5(33 \%)$ \\
\hline Academic & \\
\hline Academic/community & $2(13 \%)$ \\
Years since fellowship: & $8(54 \%)$ \\
\hline$<5$ & $2(13 \%)$ \\
\hline $5-15$ & $3(20 \%)$ \\
\hline $15-25$ & \\
\hline$>25$ &
\end{tabular}

Table 2: Discrepancies in approaches and priorities between patient and physician participants 


\section{Getting back to chemotherapy}

So once I can get this under control and everything's all right, they said we would talk back about [the trial]. (PT7)

I'm worried about how soon can I get back on track with my treatments. (PT8)

While my goal is different. My goal is to get all of this cleaned up as fast as we can, and then, let's see what we can do with the parp inhibitor. (PT5)

\section{Goals}

Prioritizing being home

I'm looking forward to just being at home. Just being home. Like I said, we've got this trip planned to go to Colorado, though I won't be hiking this time. (PT 10)

My hopes are to get home and being able to manage this with no pain. I'm still hopeful that it will resolve, but I don't know, it's been so long. (PT15)

Just taking a shower in my own shower, playing with my kids (PT9)

Oh, my God, just to sit in my living room. I mean it's not real fancy or nothing, but I just want to go home (PT8)

But I just wanted to do... you know, just regular things. Day to day routine. (PT7)

\section{End Of Life conversation}

Range of readiness

I'm not ready to end my life. I'm ready to have fun, this is my prime time. (PT7)

I don't care about my hair. I don't care about any of that. I just want to live. (PT9)

And my family's dying for me get stuff [will and advanced directives] in place. And so with this hospitalization I just realize anything can happen, and I have to do that. (PT2)

I just have to pray to God and tell him, I don't want cancer, take it away, but if I die, I die. (PT12)

I just want to really enjoy what I can. We're prepared for end of life, so to speak. (PT14)
Physician perspectives

\section{Stopping treatments}

So there's stress related to having to communicate that we have, for most of these patients the reality is that we have reached the end of the road in terms of active chemotherapy or tumor therapy. (MD10)

Most patients get to that point by being resistant to multiple chemotherapeutic agents, so it's a different situation to offer another chemotherapy with limited efficacy and a poor prognosis. But, that's also sometimes on the agenda to discuss. (MD 3)

\section{Prioritizing nutrition}

I think most patients want to get back to eating, tasting and enjoying foods. That becomes the hardest conversation if you don't think you can do that. (MD13)

I think the [gastric] tube gets placed and whether it's a partial placebo effect from the new intervention that brings with it promise and hope, or not, the patients get better, and they have also the idea that they're going to be able to have some nutrition through that. (MD1)

It's really the eating. And then the calories (MD5)
Need to talk about dying

I feel a bowel obstruction is typically a terminal event, but I guess the word terminal kind of varies it could be months, it could be weeks (MD12)

The way I try to bring it up is that "we know this is not going to be a curative situation. We do know there's a limited amount of time that you have, ... but it is important to think about what you want to do with the time that you have." (MD15)

You also can't predict completely how things are going to go, so that's really hard because you want to counsel appropriately but sometimes things don't go that way. So I think the unknown for the patient is really hard, then it makes it hard for you too. (MD15) 
They're being very diplomatic, choosing their words well. Because they realize that I realize how serious it is. I don't think they want to tell us too much either, too much in advance, because they don't know exactly. (PT5)

[My doctor] acts as though he doesn't want to talk to me about certain things that are painful. He acts as though he'd rather someone else to talk to me. [...] I'm a grown-up woman. I am 81 years old. I can take anything. (PT12)

Some of the stuff they tell you contradict themselves. Oh, we want you to eat, really keep up with your nutrition. But just little things throughout the day. But don't go do this. So, what am I supposed to do?? You know, you're going to have to follow me around and say what is what. (PT5)

\section{Communication style}

Positive thinking

I don't know how long is it going take before I see some light. It's all I can see right now, but I don't know, I'm just holding on. (PT8)

Don't give up. They say oh, well, this isn't going to work, that isn't. Don't do that. Don't stop. Keep going. Because there is a way. God is good. And He will pull out all our needs for us. So don't give up. Don't say no. (PT7)

And I trust that they have the experience not to tell me too much right now. Because I am feeling fairly optimistic for some reason, and like I say I'm not questioning it, it's there, so I'm taking advantage of that optimism to help me. (PT5)

\section{Decision-making}

Desires direction

She had mentioned this trial which I guess I'm no longer an option for. So I need to know what to do next. (PT9)

We just, we ask for our marching orders and she tells us what to do. (PT1)

I didn't write the book on cancer, I'm not a doctor. You're kind of blindsided. So you're looking for their opinion and you hope it's a good one. (PT14)

[I hope there's more than option] to choose from. I'm hoping they choose for me, to tell you the truth. I would listen to
I think that actually the majority of patients when they come in for their first malignant bowel obstruction really don't know what it means. I'm actually kind of surprised that they really don't understand how bad of a diagnosis it can be. Across the board, no matter how educated or how much experience they have the health care system, they think, I can beat this, this is overcome-able. (MD2)

I can think of one patient who was unique in that she was really direct and was not afraid to ask the next question. I think most patients, even if they get it, they're afraid to ask. (MD1)
Keeping expectations realistic

There's a misunderstanding of hope, how hope is defined and managed. And the tension between hope and reality and the importance of some degree of reality in allowing people to work through what the last months of their life mean. (MD9)

Their expectation of health outcomes were more black and white. "This is my problem, it should be a solvable problem. And solving a problem should mean that I should get back to exactly where I was before, to how I was feeling a number of years ago." And that's where it's hard. (MD2)

It's good to know that they have an expectation that's realistic. But it's hard. (MD15)

\section{Uncertainty}

Go in with an open mind into reviewing each case. I had a case in my fellowship [...] there were 3 different attendings who had 3 different opinions about her. (MD12)

You can't predict completely how things are going to go, so that's really hard because you want to counsel appropriately [...] I think the unknown for the patient is really hard, then it makes it hard for you too. (MD15)

How much does it really benefit the patient, or not. And what is the line between a palliative diversion and a surgery that will provide a much longer benefit in terms of continuing chemotherapy and so on. There's no good answer to that, we just don't know (MD10) 
their options more closely than my own gut at this point. (PT2)

That's one thing I've learned, things

change from minute to minute,

sometimes. (PT5)

Table 3: Factors that facilitate patient-physician communication

If you could predict who's going to have the horrible, Godawful life-ruining complications, it would be easier. (MD5) 


\section{Patient Perspectives \\ Trust}

I think that [my doctor] has put everything out there and has explained all the different options. I trust her with everything. We discuss everything, and then I'll tell her what I'm feeling. She'll tell me what she's feeling. Usually, we come up with the same answer. (PT10)

He was very calm and you didn't see any panic in his eyes, you didn't see like, oh, we're going to cure you in his eyes, so I trusted him to tell me the way it is now. (PT5)

That's why I listened to him because he knows of the problem of the future. (PT15)

\section{Being heard}

I think it's important for everybody to sometimes trust the patient, and especially if they've been sick for a while, to trust where they're at in their sickness. (PT2)

I think being proactive yourself was essential, without becoming... I'm very proud of not being a hypochondriac, but I've become more of a hypochondriac now, I'm more inclined to speak up for myself. (PT1)

\section{Corroboration of information}

It's been always really good that [my doctor] reaches out to other colleagues to get their input. It's not just her making decisions which I really enjoy. (PT10)

My thing is, I want to do some more research on [an ostomy] and what life is like with that routine, and maybe talk to some people who've had it actually. (PT11)

Again, I think every person owes themselves to not just be blindly led by the collar. People do have lives, even though they're surgeons or whatever they're doing for you. You have to do some of your own research and be your own advocate because they can't do it all. (PT14)
Physician perspectives

Of course it helps if it's your patient that you've been treating and it's her third or whatever number of recurrence, and you have a relationship, that always makes the conversation a lot easier. (MD10)

And a lot of it comes down to trust, it's much easier to have these conversations with someone you've followed for a really long time. They've seen some successes in their treatment, and then are now not seeing success, it's a much easier conversation. (MD2)
I guess it depends on the patient, if they're an "I like all the information" kind of patient, then, yes, but there are also some patients, who are like, "I just want to move on, I don't really need all the cerebral information, and I would rather just deal with it when it comes," so I kind of gage it. (MD12)

I really, really want them, to the extent they're interested, to verbalize what they're feeling about it. I don't usually get any Oprah-profound comments. I'm eliciting that just so that they're heard, I think that's one of the basic things about our interactions with them is that they be heard. (MD1)

\section{Time}

So when I heard that option, as the top option, I want to go home, think about the next options in my own
I think it's okay to talk to someone about it, it's not clear-cut and maybe when you review the chart you don't know the answer for a reason, it's because there's no right answer, and maybe you need someone who has done this before a few times. (MD12)

And even for me it's so difficult that I consult with my senior colleagues and try to talk with them or with my fellows, and just use them as a mirror to think if I missed something. (MD7)

I would definitely ask my partners ideas and opinions, and not take the burden of making a decision alone, because, again, with the prolonged relationship, your decision-making may not be as clear as you think it is, or may be compounded by biases that you don't even realize. (MD3)
The clinic visits we have seem so time-pressured that it's really challenging to know what really [patients] are capable of talking about, and what they're interested in talking about, because 
environment. (PT11)

Sometimes, I just need a few minutes of just taking it all and putting it on the side for a while. Not thinking about it. Then come back to it and think about it. Instead of just diving in. [laughs] So, that's how I deal with it (PT10)

I think that since this has been going on for so many years, probably the hardest lesson for me to learn is I've always been a planner, plan ahead.

Now I've learned to just wake up and see what each day brings me. (PT10) they're doing the same thing we are doing, which is making these guesstimations about what we can spare in terms of attention and focus. ... This is such a complicated one that I think those patients who do have specific concerns about it, we don't usually talk about it. (MD1)

Something I feel like, if we don't have the time, [palliative care doctors] have time, more time, to spend with the patient. (MD12)

Table 4: Communication strategies used by physicians in the setting of difficult conversations associated with MBO diagnosis 
Shared decision-making

A lot of it is about whether they have realistic understanding about what they may end up with, that they could end up with short bowel, and could end up with an ostomy or with chronic diarrhea and be IV dependent. ... And when people have said to me, I totally get it, but I'm willing to take that risk, and I change my mind. (MD9)

I usually try to put myself in their shoes and figure out what I would do. But the problem with that paradigm is that my patients are not me. But I usually do tell them what I think is best, and I tell them what we're considering, and if they feel strongly about an option that maybe I wouldn't do for myself but I think is reasonable, then I would do it. (MD12)

When somebody's looking you in the eyes and saying, "How can you not offer me this thing, why won't you give it to me?" ... I don't think it's right, but I don't think it's outside the standard of care to offer it to you, and if you want it and you understand the risks, I don't feel like it's my place to decide. (MD5)

\section{Providing options}

I give options. I let them think over the options, but then I tell them, "I'm going to come back and tell you what my preferred option is and why." ... Sometimes when you come back and you ask them, they pick the option that you'd prefer, so it saves you some time. When they don't pick the option you prefer, that takes a little more time. (MD13)

I'm probably not directive enough. I was raised in the mindset that you just need to give the patient the options and let the patient decide. And the longer I practice, the more I think a lot of patients do better with more clear recommendations or direction from the physician. (MD5)

In general, I tend to give people options. ... I do tell people that if I feel strongly about something, I'll let them know. (MD14)

\section{Directive recommendations}

I am pretty directive with what I recommend. Because I think that it is one of the patient complaints, ... "you're the doctor, I don't know how to make sense of these options, you tell me what you think." (MD2)

I tell them the options, but I do believe that the patients deserve to hear a recommendation from me. ... So I commit to something I think is the right thing to do, knowing a little bit at least their life circumstances. And it's such a difficult decision for me, that I think it's almost impossible for a patient to make an educated decision about this, it's almost impossible. (MD7)

I always make a recommendation. I usually talk to them about: here are all the options, here's why I recommend this one, and go from there. (MD4)

There's very few situations in medicine where you actually have equivalent alternatives. [...] Most of the time, you have pros and cons, and you have to weigh them for the patient. [...] I think it's our duty as doctors. You have to give them time. (MD11)

\section{Use of data}

I keep it more general. I've found that the faster I go into offering statistics, the more difficulty most patients have, I don't think most patients deal with that too well, from either a distress standpoint or the numbers helping them translate to a rational, satisfactory, not that "rational" is the goal, but a satisfactory decision for them. (MD1)

If we were to have some specific numbers, I would go back to a patient and say, there are our numbers, this is kind of where you are on the spectrum of what we've seen, and this is potentially what you could expect in terms of quality of life with some of the interventions that we've done, and how does that fit with what you think about, what your goals are. (MD2)

\section{Emphasizing risks}

I would probably offer surgery and scare the heck out of the patient by excessively talking about the risks. (MD5)

I usually counsel people that [TPN] doesn't make people feel better and that there are a lot of risks associated with doing the TPN, like infection, liver abnormalities, etc. ... And usually, people don't want it after that. I definitely don't sell it. I do the negative spin on it, that's usually how I counsel people. (MD14) 


\section{Scenarios}

If I am resolute about my recommendation, which is not all the time but is most of the time, I feel obliged at that point to be more and more detailed about how I tell them what the best and worst case scenarios are. And that leads me to this other thing that I've kind of learned in recent years, to use descriptions of scenarios as a way of communicating all that stuff. (MD1)

I think that the whole benchmarking thing sometimes will help with that as well, because they'll remember specific examples of what you said. And I have had family members come back and say: last time we were in you said that this would not be a good sign, that this is a big step back. (MD2)

Table 5: Communication Guide for in the setting of inpatient MBO diagnosis. 


\begin{tabular}{|c|c|c|}
\hline$\overline{\mathrm{IIN} *}$ & SAMPLE STATEMENTS & $\begin{array}{l}\text { THEMES } \\
\text { REFLECTED IN } \\
\text { INTERVIEWS }\end{array}$ \\
\hline \multicolumn{3}{|l|}{ al Considerations } \\
\hline $\begin{array}{l}\text { Ig: } \\
\text { Nith the patient. } \\
\text { I for family/ caregivers } \\
\text { e present in person, } \\
\text { ne or video. } \\
\text { sider recording or } \\
\text { g notes } \\
\text { id feeling rushed } \\
\text { w patient to feel } \\
\text { ritized }\end{array}$ & $\begin{array}{l}\text { I would like to discuss your current clinical } \\
\text { situation and be sure you and I are on the same } \\
\text { page. } \\
\text { Where do you want to start our discussion } \\
\text { today? }\end{array}$ & $\begin{array}{l}\text { Discrepancies (Table } \\
\text { 2) } \\
\text { 1. Paucity of } \\
\text { information vs } \\
\text { Difficulty } \\
\text { synthesizing } \\
\text { information } \\
\text { 2. Range of } \\
\text { readiness for EOL } \\
\text { conversations vs } \\
\text { Need to talk about } \\
\text { dying } \\
\text { Facilitators (Table 3) } \\
\\
\text { 1. Trust } \\
\text { 2. Time }\end{array}$ \\
\hline
\end{tabular}

ying the Diagnosis of MBO

cribe cause and

ings of $\mathrm{MBO}$

:uss overall prognosis

[BO

ress the likelihood of sistent, recurrent or yressive MBO
A malignant bowel obstruction is a sign that the cancer is getting worse [despite treatments].

Would you like to see some of the images from your CT scan?
Discrepancies (Table

2)

1. Paucity of information vs Difficulty synthesizing information

Facilitators (Table 3)

1. Corroboration of information

\section{e Awareness}

lain that there may be tiple appropriate lagement options, now as the situation nges nowledge uncertainty.
We will keep having discussions as your symptoms and priorities change throughout your care. I do think that today there are multiple considerations about what to do next for your obstruction and your cancer.
Discrepancies (Table

2)

1. Paucity of information vs Difficulty synthesizing information

2. Desires direction vs Uncertainty

Facilitators (Table 3)

1. Trust

\section{n Clarification}

cribe options under sideration.

:lear with what may not n option and why sider short-term and rer-term expectations. ride concrete and istic parameters for
There are no perfect options at this point. Everything will have risks and benefits.

Surgery is not an option because we cannot fix all the areas of blockage.

A gastric tube can help relieve nausea. It will not provide nutrition.
Discrepancies (Table 2)

1. Prioritizing being home vs Prioritizing nutrition

2. Getting back to chemotherapy 
re cancer-directed tment.

ify current symptoms discuss role of lications for nausea, el regimen, steroids if ropriate sider palliative care sultation
If you are unable to take in enough to stay hydrated, we could not give you more chemotherapy. vs Stopping treatments

3. Range of readiness for EOL

conversations vs Need to talk about dying

Facilitators (Table 3)

1. Trust

2. Corroboration of information

Communication strategies (Table 4)

1. Shared decisionmaking

2. Providing options

3. Directive recommendation

\section{Harms and Benefits Discussion}

ress expected

zomes and goals of each

on.

sider a hope-worry

ement.

sider best case/worst ? scenarios, as well as

t likely scenario, to ress uncertainty and range of possibilities.
I hope that you can travel to see your family, and I worry that after a surgery you will be more limited in your mobility and would not be able travel.

\section{Patient preferences deliberation}

it patient goals and rities.

ว evaluate how realistic ts are.

us on short term goals longer term goals. e time and space for patient to speak and be rd.
Are your pain and nausea controlled? What else is bothering you?

Who lives with you at home? How would they handle managing a wound or giving you injections? Are they able to be home whenever you need them?
Discrepancies (Table 2)

1. Positive thinking vs Keeping expectations realistic

2. Range of readiness for EOL conversations vs Need to talk about dying

3. Paucity of information vs Difficulty synthesizing information Communication strategies (Table 4)

1. Use of data

2. Emphasizing risks

3. Scenarios

Discrepancies (Table 2)

1. Prioritizing being home vs Prioritizing nutrition

2. Getting back to chemotherapy vs Stopping treatments 
ls of care conversation uding code status lld be introduced
3. Range of readiness for EOL conversations vs Need to talk about dying Facilitators (Table 3)

1. Being heard

\section{Making Decisions}

patients look towards physician for help with on-making. ude family members in scussion.

What do you think?

What further questions do you have? Discrepancies (Table 2)

1. Desires direction vs Uncertainty

*Domains adapted from Wieringa et al \{Wieringa, 2019 \# 725\}

\section{Supplementary Files}

This is a list of supplementary files associated with this preprint. Click to download.

- Suppl1interviewguides.pdf 